\section{G172 NEONATAL SEIZURES: THE UTILITY OF MAGNETIC RESONANCE IMAGING IN DIAGNOSIS AND PREDICTION OF NEURODISABILITY}

doi:10.1136/archdischild-2013-304107.184

${ }^{1} \mathrm{~A}$ Billetop, ${ }^{1} \mathrm{E}$ Osmond, ${ }^{2} \mathrm{~S}$ Jary, ${ }^{1} \mathrm{MV}$ Tsakmakis, ${ }^{1} \mathrm{~K}$ Gowda, ${ }^{3} \mathrm{M}$ Likeman, ${ }^{2} \mathrm{M}$ Thoresen, ${ }^{2} \mathrm{~K}$ Luyt. 'Neonatal Intensive Care Unit, St Michael's Hospital, Bristol, UK; ' ${ }^{2}$ School of Clinical Sciences, University of Bristol and Neonatal Intensive Care Unit, St Michael's Hospital, Bristol, UK; ${ }^{3}$ Neuroradiology, Frenchay Hospital, Bristol, UK

Aim To determine the etiological association, neurodevelopmental sequelae and role of Magnetic Resonance Imaging (MRI) in term newborn infants with seizures.

Methods Cohort study of infants $>37$ weeks gestation delivered in a single level 3 centre in the UK from 1/12/2004 to 31/10/2009 (59 months), prospectively identified by a perinatal morbidity surveillance system and followed longitudinally for 18-24 months.

Results 77 infants were identified with seizures during the study period (3.0/1000 live births). 9 infants died (12\%). 73/77 (95\%) had an aEEG recording and 70/77 (91\%) had an MRI scan

In $95 \%$ of cases an underlying cause of seizures was elucidated. The most common diagnosis was HIE (65\%) with neonatal stroke as the second most common diagnosis (12\%).

Overall, 28/77 (36\%) of the cohort had a neurodevelopmental impairment (NDI), ranging from mild to severe. $37 / 50$ infants with HIE $(74 \%)$ received therapeutic hypothermia and 9 (24\%) had NDI. Of the remaining 13 managed with normothermia, 4 had NDI (31\%). NDI was prevalent in those with a diagnosis other than HIE $(15 / 27 ; 56 \%)$, in particular stroke, hypoglycaemia and cerebral dysgenesis

$15 / 77$ (19\%) had recurrence of seizure in the first 2 years of life. The highest risk of seizure recurrence was in cerebral dysgenesis, hypoglycaemia and stroke. Infants with HIE had a very low risk of seizure recurrence $(1 / 50 ; 2 \%)$.

$45 / 70(64 \%)$ had abnormal MRI findings. The negative predictive value of a normal MRI in survivors of developing any disability was 0.93 .

Conclusion This is the first report of a cohort of term infants with seizures fully investigated by MRI and aEEG.

HIE was the most common cause of seizures in this population. Neonatal stroke was the most likely diagnosis for term infants presenting with seizures on day two of life. The universal use of MRI allows a cause to be identified in $95 \%$ of term infants with seizures. A normal MRI scan was highly predictive of a normal neurodevelopmental outcome.

There was significant risk of NDI and seizure recurrence, particularly in those with a diagnosis other than HIE, supporting formal neurological surveillance.

\section{Children's Cancer and Leukaemia Group/ British Paediatric Haematology Society}

\section{G173 DEVELOPMENT OF THE EVALUATING QUALITY OF LIFE IN ACUTE LYMPHOBLASTIC LEUKAEMIA (EQUALL) QUESTIONNAIRE: A TREATMENT SPECIFIC MEASURE FOR THE EFFECTS OF CORTICOSTEROIDS ON QUALITY OF LIFE}

doi:10.1136/archdischild-2013-304107.185

'MR Adams, 'S Sherratt, 'A Johnson, ${ }^{2} \mathrm{~J}$ Tomlins, ${ }^{3} \mathrm{~J}$ Grainger, ${ }^{1} \mathrm{MEM}$ Jenney. ${ }^{1}$ Paediatric

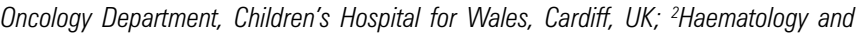
Transplant Unit, Christie Hospital NHS Trust, Manchester, UK; ${ }^{3}$ Paediatric Haematology Department, Royal Manchester Children's Hospital, Manchester, UK

Aims Survival rates for childhood ALL now exceed $80 \%$. The use of corticosteroids (particularly dexamethasone) has contributed greatly to this success. However this is not without cost - in addition to physical side effects, corticosteroids influence behaviour, mood and cognitive functioning leading to an impaired quality of life (QoL) for patients. The current UKALL 2011 trial randomises patients to maintenance therapy with or without dexamethasone pulses. Survival and QoL are primary outcome measures. The aim of this study was to develop a sensitive QoL measure that may detect potential differences in QoL in patients receiving dexamethasone.

Methods Children and young adults aged 8-24 years and parents of children aged 1-15 years receiving maintenance therapy for ALL from 4 UK centres, were invited to participate. The study comprised 3 stages: A) focus groups and interviews were conducted with participants being asked to describe their experience of dexamethasone. Version 1 of EQuALL was developed from the themes identified. B) This version was emailed in electronic survey format to healthcare professionals and patients to evaluate the importance and relevance of the questions. Amendments were made to create Version 2. Stage $\mathrm{C}$ assessed face validity and interpretation of individual questions using cognitive interviewing. Further modifications were made to define Version 3

Results Six parents and 8 patients attended focus groups/interviews. Interpretative phenomenological analysis of transcripts identified that patients feel dexamethasone has adverse effects on behaviour, appetite, body image, mood and family relationships. 121 healthcare professionals and 36 patients/parents completed the electronic survey leading to further amendments. Face validity was confirmed by cognitive interviewing of 21 patients. EQuALL now comprises 35 questions within 4 domains and has age-specific versions.

Conclusions EQuALL is the first treatment-specific QoL measure for corticosteroids. It can be completed in 10-15 minutes by children aged 8 years and above. Further validity and reliability testing will be undertaken within UKALL 2011. Although initial applications are for children with ALL, EQuALL may be a valuable clinical tool for understanding the impact of dexamethasone in other paediatric conditions.

\section{G174 TREATMENT-RELATED MYELOID LEUKAEMIA AFTER ACUTE LYMPHOBLASTIC LEUKAEMIA IN A 12 YEAR-OLD GIRL WITH BLOOM'S SYNDROME DUE TO NOVEL BLM MUTATIONS}

doi:10.1136/archdischild-2013-304107.186

${ }^{1} M R$ Adams, ${ }^{1} M$ Jenney, ${ }^{2} \mathrm{R}$ White, ${ }^{3} \mathrm{~S}$ Birdsall, ${ }^{4} \mathrm{~T}$ Staab, ${ }^{4} \mathrm{D}$ Schindler, ${ }^{5} \mathrm{~S}$ Meyer. 'Paediatric Oncology Department, Children's Hospital for Wales, Cardiff, UK, ${ }^{2}$ Department of Genetics, University Hospital of Wales, Cardiff, UK; ${ }^{3}$ Department of Haematology, University Hospital of Wales, Cardiff, UK; ${ }^{4}$ Biocenter, Biozentrum Universitat Wurzburg, Wurzburg, Germany; ${ }^{5}$ Department of Paediatric Haematology, Royal Manchester Children's Hospital, Manchester, UK

Bloom's syndrome (BS) is an inherited genome instability disorder caused by defects of the BLM helicase with extreme cancer predisposition. Here we report a girl with BS who developed acute lymphoblastic leukaemia (ALL) aged 9 and subsequently treatment-related acute myeloid leukaemia (AML) aged 12. The girl was born to a nonconsanguineous white British couple. She had intrauterine growth retardation (Bwt 1.88Kg) and was followed up during infancy for faltering growth and dysmorphic features, but no definitive diagnosis was made. At 9 years of age old she was referred with unexplained bruising. A full blood count revealed pancytopenia and blast cells consistent with ALL, which led to the diagnosis of BS three weeks into treatment. She was treated on the ALL 2003 protocol with dose modifications for toxicity. Sixteen months after completion of ALL treatment she developed an aggressive secondary AML. In view of refractory disease and extreme toxicity treatment was discontinued after two courses of chemotherapy.

BS is a DNA fragility syndrome and affected individuals have a $>50 \%$ risk of developing a malignancy. While the spectrum and 